\title{
Powerful Concentration of Rhodium in Plating Wastewater Using Homogeneous Liquid-Liquid Extraction (HoLLE) and Study for Scale-up
}

\author{
Takeshi Kato ${ }^{1}$, Shotaro Saito ${ }^{2}$, Shigekatsu Oshite ${ }^{3} \&$ Shukuro Igarashi ${ }^{2}$ \\ ${ }^{1}$ Industrial Technology Institute of Ibaraki Prefecture, Japan \\ ${ }^{2}$ Department of Biomolecular Functional Engineering, Faculty of Engineering, Ibaraki University, Japan \\ ${ }^{3}$ Department of Chemistry and Biochemistry, Fukushima National College of Technology, Japan \\ Correspondence: Takeshi Kato, Industrial Technology Institute of Ibaraki Prefecture, Ibaraki-machi, Ibaraki, \\ Japan. E-mail: katou@kougise.pref.ibaraki.jp
}

Received: July 12, 2017

Accepted: September 20, 2017 Online Published: November 27, 2017

doi:10.5539/enrr.v7n4p44

URL: https://doi.org/10.5539/enrr.v7n4p44

\begin{abstract}
A powerful technique for the concentration of rhodium $(\mathrm{Rh})$ in plating wastewater was developed. The technique entails complexing Rh with 1-(2-pyridylazo)-2-naphthol (PAN) followed by homogeneous liquid-liquid extraction (HoLLE) with Zonyl FSA. The optimum HoLLE conditions were determined as follows: $[\text { ethanol }]_{\mathrm{T}}=$ 30.0 vol. $\%, \mathrm{pH}=4.00$, and $\mathrm{Rh}: \mathrm{PAN}=1: 5$. Under these optimum conditions, $88.1 \%$ of $\mathrm{Rh}$ was extracted into the sedimented liquid phase. After phase separation, the volume ratio [aqueous phase (Va)/sedimented liquid phase $(\mathrm{Vs})]$ of $\mathrm{Va}$ and $\mathrm{Vs}$ was $1000(50 \mathrm{~mL} \rightarrow 0.050 \mathrm{~mL})$. We then applied the new method to wastewater generated by the plating industry. The phase separation was satisfactorily achieved when the volume was scaled up to 1000 $\mathrm{mL}$ of the actual wastewater; $84.7 \%$ of $\mathrm{Rh}$ was extracted into the sedimented liquid phase. After phase separation, $\mathrm{Va} / \mathrm{Vs}$ was $588(1000 \mathrm{~mL} \rightarrow 1.70 \mathrm{~mL})$.
\end{abstract}

Keywords: Homogeneous liquid-liquid extraction; HoLLE; Zonyl FSA; Rhodium; Plating wastewater

\section{Introduction}

Ruthenium ( $\mathrm{Ru}$ ), rhodium $(\mathrm{Rh})$, palladium $(\mathrm{Pd})$, osmium $(\mathrm{Os})$, iridium (Ir), and platinum (Pt) belong to the platinum group metals (PGMs), which possess superior heat and corrosion resistance properties. PGMs have been widely used in electronic devices due to their excellent physical and chemical properties. In 2013, the total world productions of Pd and Pt were 203,000 kg and 184,000 kg, while the total production of other PGMs (Ru, Rh, Os, and Ir) was $67,700 \mathrm{~kg}$ (Loferski, 2015). Because PGM production is unevenly distributed, and the prices of PGMs exhibit large fluctuation, securing PGMs is a global issue.

PGMs have been widely separated and purified using hydrometallurgical methods. Among these methods, solvent extraction is the most commonly used industrial process and is used in INCO's Acton refinery (Barnes \& Edwards, 1982). $\mathrm{Rh}$ is recovered from the residual liquid in the distillation and solvent extraction of $\mathrm{Ru}, \mathrm{Pd}, \mathrm{Os}$, Ir, and $\mathrm{Pt}$ owing to the most difficult step in the extraction of PGMs (Tanaka, Koyama, Narita, \& Oishi, 2011). This is because $\mathrm{Rh}$ ions are more strongly hydrated than other PGMs; thus, Rh exhibits extreme inertness during the extraction process (Yan \& Alstad, 1995). As a soft acid, Rh can be selectively extracted using soft donor base with nitrogen or sulfur atoms (Kumar, Lee, Kim, \& Sohn, 2009; Shaikh, Lande, \& Arbad, 2011). A variety of nitrogen-based extractants, such as alamine 304-1 (Sun, J.Y. Lee, \& M.S. Lee, 2011), tri-octylamine (Jaree \& Khunphakdee, 2011), and N-decylpyridine-4-amine (Bagal, Sakhare, Lande, \& Arbad, 2014), and a variety of sulfur-based extractants such as dialkyl sulfoxides (Preston \& Preez, 2002) and trialkylphosphine sulphide (Rizvi, Mathur, Murali, \& Iyer, 1996), have been investigated for Rh extraction.

Although solvent extraction is a useful separation and purification method, it has several drawbacks related to operability and efficiency. In general, solvent extraction requires mechanical shaking to increase the surface area between the aqueous and water-immiscible organic solvent phases. In contrast, in homogeneous liquid-liquid extraction (HoLLE), no interface exists between the aqueous and water-immiscible organic solvent phases; that is, the initial condition is a homogenous solution (Igarashi \& Yotsuyanagi, 1988). The HoLLE procedure is 
straightforward and only requires the addition of reagents due to the large surface area of the interface. In HoLLE, the target solute is concentrated into a small secondary phase through the effect of some stimulation such as $\mathrm{pH}$, or light. HoLLE has been widely used to extract target solutes such as indium (In) (Kato, Igarashi, Ishiwatari, Furukawa, \& Yamaguchi, 2013), cobalt (Co) (Kato, Igarashi, Saito, Ando, \& Asano, 2014), rare earth elements (Saito, Ohno, Igarashi, Kato, \& Yamaguchi, 2015), and Pd (Kato, Igarashi, Ohno, Saito, \& Ando, 2016), although the target solute is not limited to metals. Additionally, HoLLE can be applied to organic compounds (Takagai \& Igarashi, 2002) and biological materials (Sudo \& Igarashi, 1996). With respect to trace amounts of metals, nanofiltration (Otero-Fernandez et al., 2017) and solid-phase extraction (Molaei, Bagheri, Asgharinezhad, Ebrahimzadeh, Shamsipur, 2017) have been reported. However, there is still room for improvement in contact probability, and interfacial area, and other factors. These problems can be solved using HoLLE because the initial condition is a homogeneous solution and the surface area of the interface is infinitely large. On the other hand, Murata and Ikeda studied the high solubility of propylene carbonate in water at high temperature as homogeneous liquid-liquid extraction (Murata \& Ikeda, 1969). In similar studies, some ionic liquid solutions form one homogeneous phase above the upper critical solution temperature or below a lower critical solution temperature (Lamb \& Peterson, 1995; Mori et al., 2015; Hoogerstraete, Onghena, \& Binnemans, 2013; Depuydt, Liu, Glorieux, Dehaen, \& Binnemans, 2015). Such homogeneous liquid-liquid extraction was applied to the extraction of metal ions (Mori et al., 2015; Hoogerstraete, Onghena, \& Binnemans, 2013; Depuydt, Liu, Glorieux, Dehaen, \& Binnemans, 2015). Compared with homogeneous liquid-liquid extraction based on temperature, HoLLE based on $\mathrm{pH}$-dependent phase separation can be used to achieve 100-100,000-fold concentration in several minutes down to micro-volume levels (Takagai \& Igarashi, 2002). For this reason, HoLLE based on $\mathrm{pH}$-dependent phase separation was investigated.

In the treatment of wastewater from the plating industry, the volume of wastewater is often reduced by heating, evaporation, and other processes because it is difficult to treat the wastewater at own facility in plating industry. Thus, HoLLE represents a promising strategy to concentrate Rh from plating wastewater. Herein, we used 1-(2-pyridylazo)-2-naphthol (PAN) as a soft donor base containing nitrogen to extract $\mathrm{Rh}$ (Cheng \& Bray, 1955; Stokely \& Jacobs, 1963). In this study, Zonyl FSA was used as a phase separation agent with an acid dissociation constant (pKa) of approximately 6 (Sudo \& Igarashi, 1996). It introduced a methylene chain as a spacer between the perfluoroalkyl group and carboxyl group in order to negate the effect of fluorine. Consequently, this method can apply complexation at mild $\mathrm{pH}$ because phase separation below $\mathrm{pH} 6$ is possible.

\section{Experimental}

\subsection{Reagents and Materials}

$\mathrm{Rh}$ plating water comprising rhodium sulfate $(0.2 \mathrm{wt} \%)$, sulfuric acid (4 $\mathrm{wt} \%)$, phosphoric acid (1.4 wt $\%$ ), and water was obtained from Ibaraki Plating Kogyo Co., Ltd. (Ibaraki, Japan). The Rh concentration of the real plating wastewater was determined as $708 \mathrm{mg} \mathrm{L}^{-1}$ using inductively coupled plasma optical emission spectrometry (ICP-OES). Aqueous solutions of metal ions were prepared by diluting $1000 \mathrm{mg} \mathrm{L}^{-1}$ standard solutions obtained from Wako Pure Chemical Industries, Ltd. (Osaka, Japan). A solution of Zonyl FSA $\left[\mathrm{CF}_{3}\left(\mathrm{CF}_{2}\right)_{\mathrm{n}} \mathrm{CH}_{2} \mathrm{CH}_{2} \mathrm{SCH}_{2} \mathrm{CH}_{2} \mathrm{CO}_{2} \mathrm{H} ; \mathrm{n}=6-8\right.$; DuPont, Tokyo, Japan] was prepared by diluting pure Zonyl FSA with an equivalent amount of distilled water. All chemicals except Zonyl FSA were obtained from Wako Pure Chemical Industries, Ltd. (Osaka, Japan). All reagents used in this study were of analytical grade.

\subsection{Apparatus}

The following instruments were employed in this study: an M-12 pH meter manufactured by Horiba (Kyoto, Japan); an AS185 centrifugal separator manufactured by AS ONE Corporation (Osaka, Japan); a U-4100 UV-VIS spectrophotometer manufactured by Hitachi High-Technologies Corporation (Tokyo, Japan); and an ICP ULTIMA2 ICP-OES manufactured by Horiba (Kyoto, Japan).

\subsection{Methods}

\subsubsection{HoLLE of a pure Rh solution}

$\mathrm{Rh}$ standard solution $\left(1,000 \mathrm{mg} \mathrm{L}^{-1}\right)$ was added to a $50-\mathrm{mL}$ centrifuge tube such that $0.1 \mathrm{mg}$ of $\mathrm{Rh}$ was added to the tube. PAN solution $(5 \mathrm{~mL}, 0.025 \%)$ and ethanol $(2 \mathrm{~mL})$ were then added to the centrifuge tube, and the volume was adjusted to $30 \mathrm{~mL}$ with phthalate $\mathrm{pH}$ standard solution ( $\mathrm{pH} 4)$. The solution was heated for $60 \mathrm{~min}$ at $90^{\circ} \mathrm{C}$ to form Rh-PAN complexes and then allowed to rest for $60 \mathrm{~min}$ at room temperature. Subsequently, phthalate $\mathrm{pH}$ standard solution $(11 \mathrm{~mL})$ and ethanol $(8 \mathrm{~mL})$ were added to the centrifuge tube, followed by the addition of $50 \mathrm{v} / \mathrm{v} \%$ Zonyl FSA $(1 \mathrm{~mL})$. After shaking gently, the solution was centrifuged at 2,500 rpm for $30 \mathrm{~min}$ in order to hasten phase 
separation. After phase separation, the sedimented liquid phase was collected using a microsyringe. The volume of the sedimented liquid phase was determined using a $0.1-\mathrm{mL}$ microsyringe.

\subsubsection{HoLLE of Real Rh Plating Wastewater}

Real Rh plating wastewater was added to a $50-\mathrm{mL}$ centrifuge tube such that $0.1 \mathrm{mg}$ of $\mathrm{Rh}$ was added to the tube. PAN solution $(5 \mathrm{~mL}, 0.025 \%)$ and ethanol $(2 \mathrm{~mL})$ were then added to the centrifuge tube, and the volume was adjusted to $30 \mathrm{~mL}$ with phthalate $\mathrm{pH}$ standard solution $(\mathrm{pH} 4)$ and small amounts of 5-M nitric acid to adjust the $\mathrm{pH}$ to 4.0. The solution was heated for $180 \mathrm{~min}$ at $90{ }^{\circ} \mathrm{C}$ to form Rh-PAN complexes and allowed to rest for $60 \mathrm{~min}$ at room temperature. Then, phthalate $\mathrm{pH}$ standard solution $(11 \mathrm{~mL})$ and ethanol $(8 \mathrm{~mL})$ were added to the centrifuge tube, followed by the addition of $50 \mathrm{v} / \mathrm{v} \%$ Zonyl FSA $(1 \mathrm{~mL})$. After shaking gently, the solution was centrifuged at $2,500 \mathrm{rpm}$ for $30 \mathrm{~min}$ in order to hasten phase separation. After phase separation, the sedimented liquid phase was collected using a microsyringe. The volume of the sedimented liquid phase was determined using a $0.1-\mathrm{mL}$ microsyringe.

\subsubsection{HoLLE for Scale-Up to Real Rh Plating Wastewater}

Real Rh plating wastewater was added to a beaker such that $2 \mathrm{mg}$ of $\mathrm{Rh}$ was added to the beaker. PAN solution $(100 \mathrm{~mL}, 0.025 \%)$ and ethanol $(40 \mathrm{~mL})$ were then added to the beaker, and the volume was adjusted to $600 \mathrm{~mL}$ with phthalate $\mathrm{pH}$ standard solution $(\mathrm{pH} 4)$ and small amounts of 5-M nitric acid to adjust the $\mathrm{pH}$ to 4.0 . The solution was heated for $180 \mathrm{~min}$ at $90{ }^{\circ} \mathrm{C}$ to form Rh-PAN complexes and allowed to rest for 60 min at room temperature. The solution was moved from the beaker to the plastic vessel. Next, phthalate $\mathrm{pH}$ standard solution $(220 \mathrm{~mL})$ and ethanol $(160 \mathrm{~mL})$ were added to the plastic vessel, followed by the addition of $50 \mathrm{v} / \mathrm{v} \%$ Zonyl FSA $(20 \mathrm{~mL})$. After shaking gently, the solution stood for about one hour. After phase separation, the sedimented liquid phase was collected by opening below cock of the plastic vessel. The volume of the sedimented liquid phase was determined using a 20 -mL measuring glass.

\section{Results and Discussion}

\subsection{Rh-PAN Complexes}

Cheng and Bray first studied the use of PAN as an analytical reagent (Cheng \& Bray, 1955). Stokely and Jacobs investigated Rh complexation with PAN for a spectrophotometric determination of Rh (Stokely \& Jacobs, 1963). Rh-PAN complexes are easily prepared by heating in buffered, aqueous-alcoholic solutions. Because it is difficult to treat organic solvent as chloroform in a plating industry, ethanol is suitable in order to require environmental conscious system. Considering Rh-PAN complexation in buffered, aqueous-alcoholic solutions, PAN was selected in this study. Upon heating, the orange solution of Rh and PAN gradually became green, suggesting the formation of Rh-PAN complexes. Figure 1 shows the absorbance curves for the Rh-PAN complexes; the Rh concentration was proportional to the absorbance of the Rh-PAN complexes. The Rh-PAN complex had an absorbance maximum at $587 \mathrm{~nm}$. On the basis of continuous variation method in absorbance at $587 \mathrm{~nm}$, the $\mathrm{Rh}: \mathrm{PAN}$ ratio was 1:1 (Figure 2).

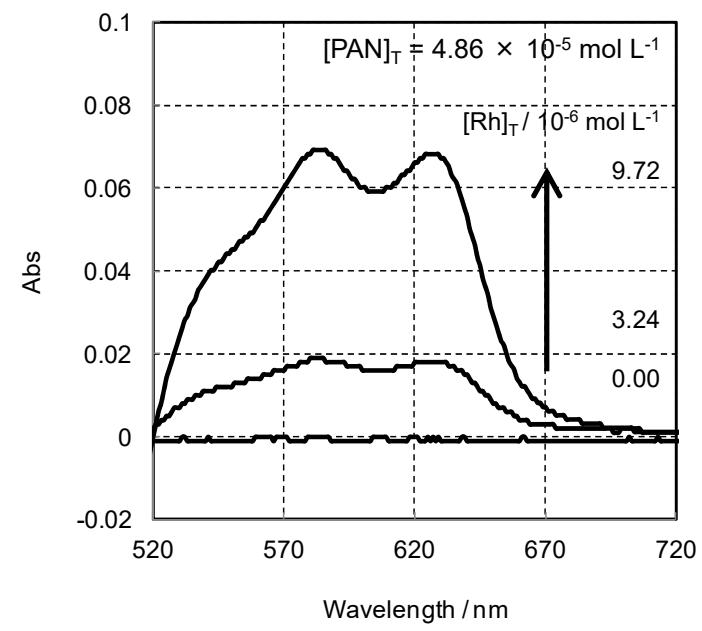

Figure 1. Absorbance curves for the rhodium(Rh)-1-(2-pyridylazo)-2-naphthol(PAN) complex. Complexing condition: $90{ }^{\circ} \mathrm{C}, 60 \mathrm{~min} ., \mathrm{pH}$ 4.01

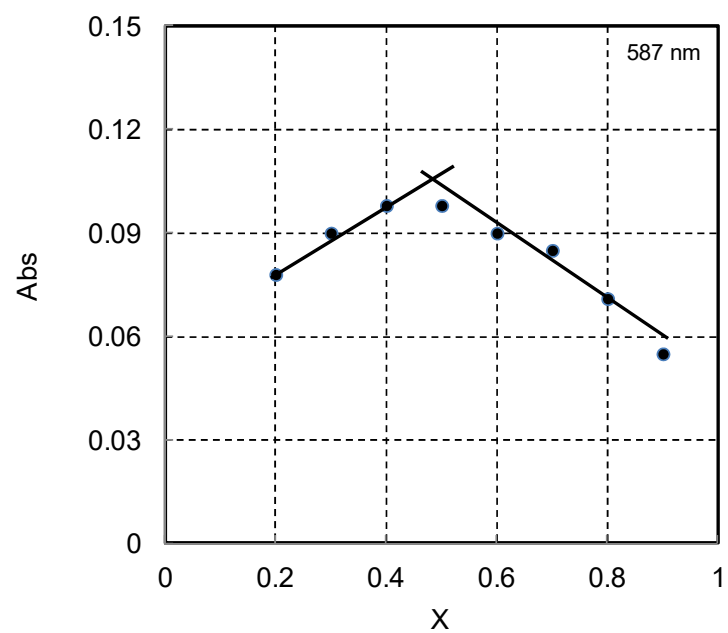

Figure 2. Continuous variation method in absorbance at 587 nm. M + nX $\rightarrow$ MXn, M: Rhodium, X: PAN, M: (1$\mathrm{x}) \mathrm{mL}, \mathrm{X}: \mathrm{x} \mathrm{mL},[\mathrm{Rh}]=[\mathrm{PAN}]=1.00 \times 10^{-3} \mathrm{~mol} \mathrm{~L}^{-1}$, Total volume: $30 \mathrm{~mL}$ 


\subsection{HoLLE of Rh}

HoLLE using a phase separation agent such as Zonyl FSA was reversible with the $\mathrm{pH}$ change in the solution, corresponding to below and above the pKa of phase separation agent such as Zonyl FSA (Igarashi \& Yotsuyanagi, 1992). If the $\mathrm{pH}$ of the aqueous solution containing small amounts of a water-miscible organic solvent such as ethanol is lower than the acid dissociation constant of Zonyl FSA ( $\mathrm{pKa}=6.5)$ (Sudo \& Igarashi, 1996), the target solute is concentrated into the sedimented liquid phase. In this study, we confirmed the behavior of HoLLE in a pure Rh solution. In particular, HoLLE using Zonyl FSA containing Rh-PAN complexes in weakly acidic media was examined. Phase separation was satisfactorily completed via HoLLE. The volume ratio, defined as volume ratio $=(\mathrm{mL}$ of the aqueous phase $) /(\mathrm{mL}$ of the sedimented liquid phase $)$, was $50 / 0.085=588$. Therefore, we concluded that HoLLE can be used to concentrate $\mathrm{Rh}$ with a high volume ratio. The analysis of the aqueous phase using ICP-OES indicated that $74.0 \%$ of $\mathrm{Rh}$ was extracted into the sedimented liquid phase. It has been reported that the water content of the sedimented liquid phase was 5-\% in the Zonyl FSA system (Sudo \& Igarashi, 1996). Based on the low-water content of the sedimented liquid phase, the hydrophobic solute as Rh-PAN complex could be effectively extracted.

After confirming the ability of HoLLE to separate solutions containing Rh-PAN complexes, we optimized the experimental conditions. First, the effect of ethanol (the water-soluble solvent) on HoLLE was evaluated because ethanol affects the state of the sedimented liquid phase. The relation between the volume of the sedimented liquid phase and the ethanol concentration was linear for ethanol concentrations ranging from $18.0 \mathrm{vol} . \%-30.0 \mathrm{vol} . \%$. At ethanol concentrations exceeding 30.0 vol.\%, the volume of the sedimented liquid phase remained constant (Figure 3). Therefore, an ethanol concentration of 30.0 vol.\% was selected for the remaining experiments. Then, we evaluated the effect of $\mathrm{pH}$ on HoLLE because $\mathrm{pH}$ contributes to Rh-PAN complexation and phase separation. Results state that the extraction percentage increased with increasing $\mathrm{pH}$ and exceeded more than $80.0 \%$ at $\mathrm{pH}$ values above 4.00. Moreover, the volume ratio was maximized at $\mathrm{pH} 4.00$. Due to increasing water content in the sedimented liquid phase, the volume ratio at $\mathrm{pH} 4.50$ was smaller than that at $\mathrm{pH} 4.00$. Huang et al. found that surfactants can form vesicles in the ethanol-water-mixed solvents (Huang, Zhu, Zhao, \& Zhang, 1997). Moreover, Lee et al. reported that surfactants can form vesicles at intermediate $\mathrm{pH}$ values close to the $\mathrm{pKa}$ of the molecules (Lee, Danino, \& Raghavan, 2009). Based on these reports, it is considered that the water content of the sedimented liquid phase increased because Zonyl FSA forms vesicles at $\mathrm{pH}$ values close to the $\mathrm{pKa}$. Thus, to obtain the optimum extraction percentage and volume ratio, a $\mathrm{pH}$ of 4.00 was chosen for the remaining experiments (Figure 4). Finally, we evaluated the effect of PAN concentration on Rh extraction. When the Rh:PAN ratio was less than $1: 3$, the extraction percentage of $\mathrm{Rh}$ was approximately $70.0 \%$; however, at a ratio of $1: 5$, the extraction percentage was $88.1 \%$ (Figure 5). Based on these results, the Rh:PAN ratio was set at 1:5 in further experiments. Using the above optimum conditions for HoLLE, the volume ratio was calculated as follows:

Volume ratio $=(\mathrm{mL}$ of the aqueous phase $) /(\mathrm{mL}$ of the sedimented liquid phase $)$

$=50 / 0.050=1000$.

Under the optimized conditions, $88.1 \%$ of Rh was extracted into the sedimented liquid phase, as determined by the ICP-OES analysis of the aqueous phase.

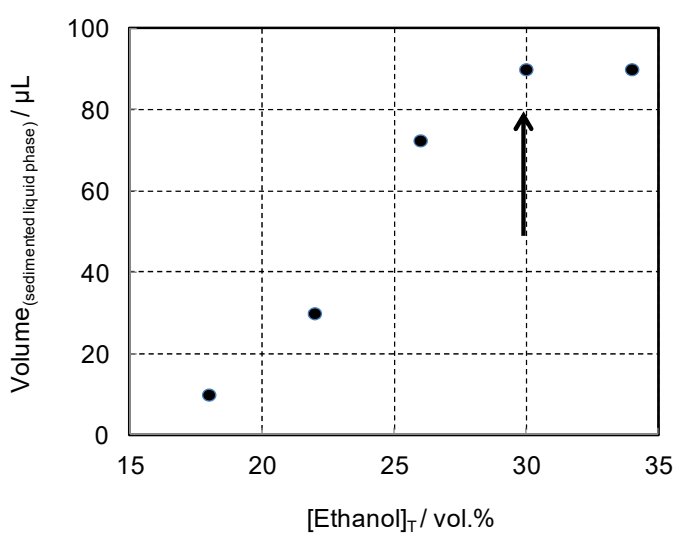

Figure 3. Effect of ethanol concentration on the HoLLE of Rh. $[\mathrm{Rh}]_{\mathrm{T}}=1.94 \times 10^{-5} \mathrm{~mol} \mathrm{~L}^{-1},[\mathrm{PAN}]_{\mathrm{T}}=$ $1.00 \times 10^{-4} \mathrm{~mol} \mathrm{~L}^{-1}, \mathrm{pH} 4.01$, [Zonyl FSA $]_{\mathrm{T}}=1.00$ vol.\%

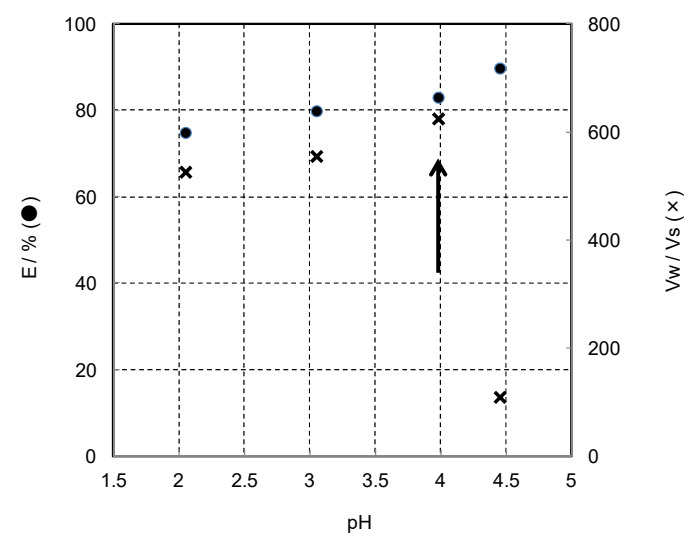

Figure 4. Effect of $\mathrm{pH}$ on the HoLLE of Rh. $[\mathrm{Rh}]_{\mathrm{T}}=$ $1.94 \times 10^{-5} \mathrm{~mol} \mathrm{~L}^{-1},[\mathrm{PAN}]_{\mathrm{T}}=1.00 \times 10^{-4} \mathrm{~mol} \mathrm{~L}^{-1}$, $[\text { Ethanol }]_{\mathrm{T}}=30.0$ vol. $\%$, $[\text { Zonyl FSA }]_{\mathrm{T}}=1.00 \mathrm{vol} . \%$ 


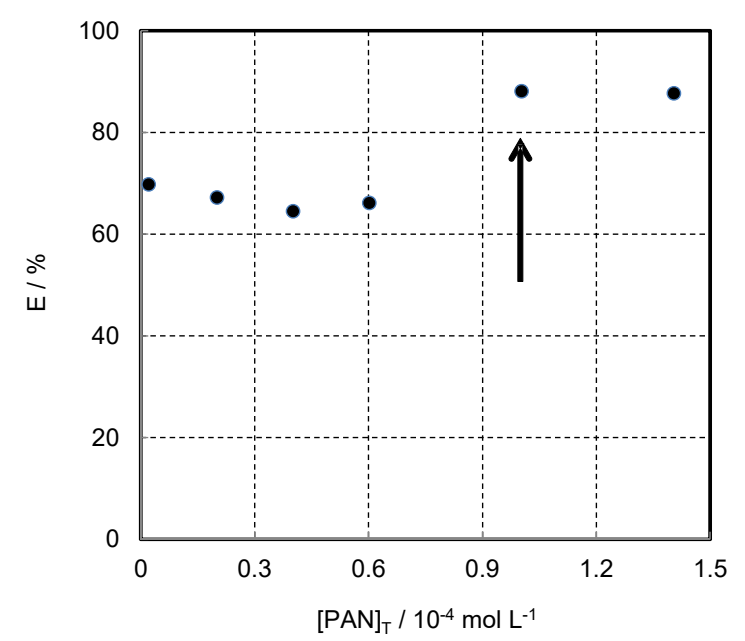

Figure 5. Effect of PAN concentration on the HoLLE of Rh. $[\mathrm{Rh}]_{\mathrm{T}}=1.94 \times 10^{-5} \mathrm{~mol} \mathrm{~L}^{-1}, \mathrm{pH} 4.01$, [Ethanol $]_{\mathrm{T}}=$ 30.0 vol. $\%$, [Zonyl FSA $]_{\mathrm{T}}=1.00$ vol. $\%$

\subsection{Scale-Up to Real Rh Plating Wastewater}

HoLLE was first performed on real Rh plating wastewater using the same volume as above to confirm the applicability of this method to actual wastewater. The results indicated that phase separation was satisfactorily achieved (Figure 6). The volume ratio was calculated as follows:

Volume ratio $=(\mathrm{mL}$ of the aqueous phase $) /(\mathrm{mL}$ of the sedimented liquid phase $)$

$=50 / 0.075=667$.

The ICP-OES analysis of the aqueous phase revealed that $86.4 \%$ of $\mathrm{Rh}$ was extracted into the sedimented liquid phase.

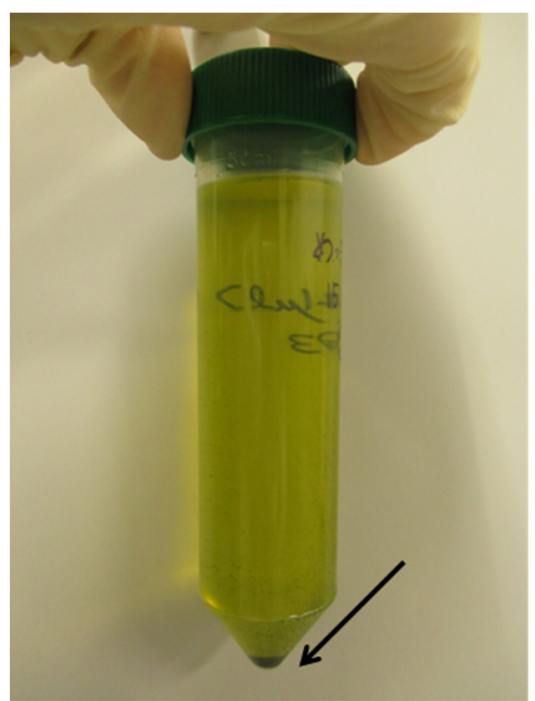

Figure 6. Solutions obtained after the HoLLE of real Rh plating wastewater. $[\mathrm{Rh}]_{\mathrm{T}}=1.94 \times 10^{-5} \mathrm{~mol} \mathrm{~L}^{-1},[\mathrm{PAN}]_{\mathrm{T}}=$ $1.00 \times 10^{-4} \mathrm{~mol} \mathrm{~L}^{-1}, \mathrm{pH} 4.01,[\text { Ethanol }]_{\mathrm{T}}=30.0 \mathrm{vol} . \%,[\text { Zonyl FSA }]_{\mathrm{T}}=1.00 \mathrm{vol} . \%$

We evaluated the potential of this method to scale-up to realistic volumes of actual plating wastewater. The total volume was increased to $1000 \mathrm{~mL}$ ( 20 times higher than the original volume), and the experimental conditions were the optimized conditions detailed above. The experiment was performed using a plastic vessel suitable in separation for treatment of several liters (Figure 7). Phase separation began in several minutes after the solution was added and mixed. Subsequently, a dark green sedimented liquid phase was formed, similar to that obtained in the above experiments. Phase separation was nearly completed in $1 \mathrm{~h}$, and the volume ratio was calculated as follows: 
Volume ratio $=(\mathrm{mL}$ of the aqueous phase $) /(\mathrm{mL}$ of the sedimented liquid phase $)$

$=1000 / 1.70=588$.

The ICP-OES analysis of the aqueous phase indicated that $84.7 \%$ of Rh was extracted into the sedimented liquid phase. Thus, HoLLE can be used for the concentration of $\mathrm{Rh}$ in realistic volumes of plating wastewater.

Perfluoro compounds, such as Zonyl FSA, can be removed with back-extraction by adding solvent (Takagai \& Igarashi, 2003), recovery using a chemically modified polymer gel (Butsugan \& Igarashi, 2009), removal by solid-phase extraction (Japanese Standard Association, 2011), and reuse on the basis of the mixed solution (Saito, Ohno, Igarashi, Kato, \& Yamaguchi, 2015). For example, rhodium leads to the formation of hydroxide, Zonyl FSA is recovered by a chemically modified polymer gel (Butsugan \& Igarashi, 2009), and PAN remains in residual liquid after the Zonyl FSA of the sedimented liquid phase is dissolved in water at a higher $\mathrm{pH}$ value than its acid dissociation constant ( $\mathrm{pKa}=6.5)$ (Sudo \& Igarashi, 1996).

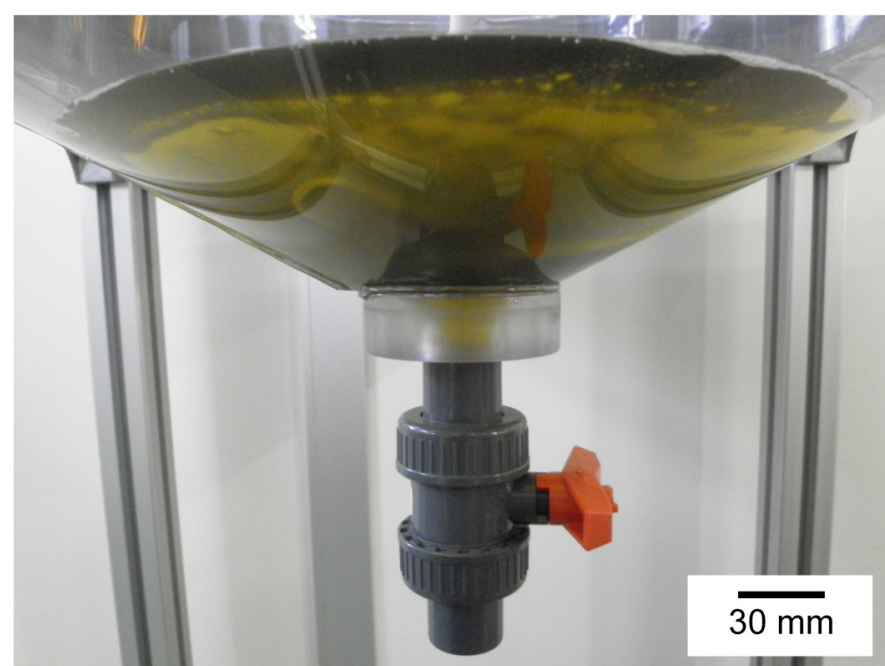

Figure 7. Plastic vessel used for the scaled-up experiment. $[\mathrm{Rh}]_{\mathrm{T}}=1.94 \times 10^{-5} \mathrm{~mol} \mathrm{~L}^{-1}$, $[\mathrm{PAN}]_{\mathrm{T}}=1.00 \times 10^{-4} \mathrm{~mol} \mathrm{~L}^{-1}, \mathrm{pH} 4.01,[\text { Ethanol }]_{\mathrm{T}}=30.0 \mathrm{vol} . \%,[\text { Zonyl FSA }]_{\mathrm{T}}=1.00 \mathrm{vol} . \%$

\section{Conclusions}

We successfully demonstrated the concentration of Rh. Under the optimized conditions, the extraction percentage was $88.1 \%$ and the volume ratio was $1,000(50 \mathrm{~mL} \rightarrow 0.050 \mathrm{~mL})$. We evaluated the potential of this method to scale-up using real plating wastewater; the results indicated that phase separation was satisfactorily completed with an extraction percentage of $84.7 \%$ and a volume ratio of $588(1000 \mathrm{~mL} \rightarrow 1.70 \mathrm{~mL})$. These findings are expected to facilitate the development of new concentration techniques to extract rare metals from plating wastewater.

\section{Acknowledgments}

These results are related to special power supply location prefecture technology promotion work by the Ministry of Education, Culture, Sports, Science and Technology, Japan. The authors would like to express their appreciation to Ibaraki Plating Kogyo Co., Ltd. (Ibaraki, Japan) for providing real rhodium plating wastewater. The authors would like to express their appreciation to Tanaka Kikinzoku Kogyo K. K. (Tokyo, Japan) for advising this study. The authors would like to thank Masae Miyakawa for assistance with the whole experiments. The authors would like to thank Enago (www.enago.jp) for the English language review. Finally, the authors would like to thank the reviewers and the editor for their time spent on reviewing the manuscript and their comments helping us in improving the article.

\section{References}

Bagal, M. R., Sakhare, M. A., Lande, M. K., \& Arbad, B. R. (2014). Liquid-liquid extraction of Rh(III) with new extractant N-decylpyridine-4-amine from malonate media. Inter. J. Anal. Bioanal. Chem., 4(1), 1-6.

Barnes, J. E., \& Edwards, J. D. (1982). Solvent extraction at Inco's Acton precious metal refinery. Chem. Ind., 5, $151-155$. 
Butsugan, M., \& Igarashi, S. (2009). Collection and recovery agent for surfactant. Japanese Patent. 207794. (in Japanese).

Cheng K. L., \& Bray, R. H. (1955). 1-(2-Pyridylazo)-2-naphthol as a possible analytical reagent. Anal. Chem., 27(5), 782-785. https://doi.org/10.1021/ac60101a024

Depuydt, D., Liu, L., Glorieux, C., Dehaen, W., \& Binnemans, K. (2015). Homogeneous liquid-liquid extraction of metal ions with non-fluorinated bis(2-ethylhexyl)phosphate ionic liquids having a lower critical solution temperature in combination with water. Chem. Commun., 51, 14183-14186. https://doi.org/10.1039/ $\mathrm{c} 5 \mathrm{cc} 05649 \mathrm{~g}$

Hoogerstraete, T. V., Onghena, B., \& Binnemans, K. (2013). Homogeneous liquid-liquid extraction of metal ions with a functionalized ionic liquid. J. Phys. Chem. Lett., 4, 1659-1663. https://doi.org/10.1021/ jz4005366

Huang, J. B., Zhu, B. Y., Zhao, G. X., \& Zhang, Z. Y. (1997). Vesicle formation of a 1:1 catanionic surfactant mixture in ethanol solution. Langmuir., 13, 5759-5761. https://doi.org/10.1021/la9610031

Igarashi, S., \& Yotsuyanagi, T. (1988). A novel homogeneous liquid-liquid extraction by $\mathrm{pH}$ dependent phase separation with fluorocarbon ionic surfactant. Proceedings of the Symposium on Solvent Extraction 1988 (pp. 175-180). Tokyo, Japan.

Igarashi, S., \& Yotsuyanagi, T. (1992). Homogeneous liquid-liquid extraction by $\mathrm{pH}$ dependent phase separation with a fluorocarbon ionic surfactant and its application to the preconcentration of porphyrin compounds. Microchim. Acta., 106(1), 37-44. https://doi.org/10.1007/bf01242697

Japanese Standard Association. (2011). Testing methods for perfluorooctanesulfonate (PFOS) and perfluorooctanoate (PFOA) in industrial water and wastewater (JIS K0450-70-10). (in Japanese).

Jaree, A., Khunphakdee, N. (2011). Separation of concentrated platinum(IV) and rhodium(III) in acidic chloride solution via liquid-liquid extraction using tri-octylamine. J. Ind. Eng. Chem., 17(2), 243-247. https://doi.org/10.1016/j.jiec.2011.02.013

Kato, T., Igarashi, S., Ishiwatari, Y., Furukawa, M., \& Yamaguchi, H. (2013). Separation and concentration of indium from a liquid crystal display via homogeneous liquid-liquid extraction. Hydrometallurgy., 137, 148-155. https://doi.org/10.1016/j.hydromet.2013.06.004

Kato, T., Igarashi, S., Ohno, O., Saito, S., \& Ando R. (2016). Homogeneous liquid-liquid extraction (HoLLE) of palladium in real plating wastewater for recovery. J. Environ. Prot., 7(2), 277-286. https://doi.org/10. 4236/jep.2016.72024

Kato, T., Igarashi, S., Saito, S., Ando, R., \& Asano, T. (2014). Development of rare metal concentration method using homogeneous liquid-liquid extraction (HoLLE) as an example for cobalt plating rinse water. J. Surf. Finish. Soc. Jpn., 65(3), 144-146. (in Japanese). https://doi.org/10.4139/sfj.65.144

Kumar, J. R., Lee, J. S., Kim, J. S., \& Sohn, J. S. (2009). Liquid-liquid extraction of platinum from acidic solutions - A review. Solvent Extr. Res. Dev., Jpn., 16, 13-22.

Lamb, J. D., \& Peterson, R. T. (1995). Coalescence extraction: a novel, rapid means of performing solvent extractions. Separ. Sci. Tech., 30(17), 3237-3244. https://doi.org/10.1080/01496399508013141

Lee, J. H., Danino, D., \& Raghavan, S. R. (2009). Polymerizable vesicles based on a single-tailed fatty acid surfactant: a simple route to robust nanocontainers. Langmuir., 25, 1566-1571. https://doi.org/10.1021/ la $802373 \mathrm{j}$

Loferski, P. J. (2015). 2013 Minerals Yearbook (pp. 1-11). U.S. Geological Survey: USA.

Molaei, K., Bagheri, H., Asgharinezhad, A. A., Ebrahimzadeh, H., \& Shamsipur, M. (2017). $\mathrm{SiO}_{2}$-coated magnetic graphene oxide modified with polypyrrole-polythiophene: A novel and efficient nanocomposite for solid phase extraction of trace amounts of heavy metals. Talanta., 167(5), 607-616. https://doi.org/10. 1016/j.talanta.2017.02.066

Mori, T., Takao, K., Sasaki, K., Suzuki, T., Arai, T., \& Ikeda, Y. (2015). Homogeneous liquid-liquid extraction of $\mathrm{U}(\mathrm{VI})$ from $\mathrm{HNO}_{3}$ aqueous solution to betainium bis(trifluoromethylsulfonyl)imide ionic liquid and recovery of extracted U(VI). Sep. Purif. Technol. 155(11), 133-138. https://doi.org/10.1016/j.seppur. 2015.01.045 
Murata, K., \& Ikeda, S. (1969). Homogeneous liquid-liquid extraction method. Bunseki Kagaku. 18, 1137. (in Japanese). https://doi.org/10.2116/bunsekikagaku.18.1137

Otero-Fernandez, A., Otero, J. A., Maroto, A., Carmona, J., Palacio, L., Pradanos, P., \& Hernandez, A. (2017). Concentration-polarization in nanofiltration of low concentration $\mathrm{Cr}(\mathrm{VI})$ aqueous solutions. Effect of operative conditions on retention. J. Clean. Prod., 150(5), 243-252. https://doi.org/10.1016/j.jclepro. 2017.03.014

Preston, J. S., \& Preez, A. C. (2002). Solvent extraction of platinum-group metals from hydrochloric acid solutions by dialkyl sulphoxides. Solvent Extr. Ion Exch., 20(3), 359-374. https://doi.org/10.1081/ sei-120004810

Rizvi, G. H., Mathur, J. N., Murali, M. S., \& Iyer, R. H. (1996). Recovery of fission product Palladium from acidic high level waste solutions. Sep. Sci. Tech., 31(13), 1805-1816. https://doi.org/10.1080/01496399608001011

Saito, S., Ohno, O., Igarashi, S., Kato, T., \& Yamaguchi, H. (2015). Separation and recycling for rare earth elements by homogeneous liquid-liquid extraction (HoLLE) using a pH-responsive fluorine-based surfactant. Metals., 5(3), 1543-1552. https://doi.org/10.3390/met5031543

Shaikh, U. K., Lande, M. K., \& Arbad, B. R. (2011). Solvent extraction separation of Rhodium(III) with 4-(4-ethoxybenzylidene amino)-5-methyl-4H-1,2,4-triazole-3-thiol (EBIMTT) as an extractant. Adv. Appl. Sci. Res., 2(6), 347-353.

Stokely, J. R., \& Jacobs, W. D. (1963). Simultaneous spectrophotometric determination of rhodium and iridium with 1-(2-Pyridylazo)-2-naphthol. Anal. Chem., 35(2), 149-152. https://doi.org/10.1021/ac60195a013

Sudo, T., \& Igarashi, S. (1996). Homogeneous liquid-liquid extraction method for spectrofluorimetric determination of chlorophyll a. Talanta., 43(2), 233-237. https://doi.org/10.1016/0039-9140(95)01748-8

Sun, P. P., Lee, J. Y., \& Lee, M. S. (2011). Separation of Pt(IV) and Rh(III) from chloride solution by solvent extraction with amine and neutral extractants. Mater. Trans., 52(11), 2071-2076. https://doi.org/10.2320/ matertrans.m2011159

Takagai, Y., \& Igarashi, S. (2002). Homogeneous liquid-liquid extraction and micellar electrokinetic chromatography using sweeping effect concentration system for determination of trace amounts of several polycyclic aromatic hydrocarbons. Anal. Bioanal. Chem., 373(1), 87-92. https://doi.org/10.1007/s00216 $-002-1243-9$

Takagai, Y., \& Igarashi, S. (2003). Selective extraction and isolation of vitamin B12 using homogeneous liquid-liquid extraction with perfluoro surfactant. Bull. Chem. Soc. Jpn., 76, 1595-1600. https://doi.org/10.1246/bcsj.76.1595

Tanaka, M., Koyama, K., Narita, H., \& Oishi, T. (2011). Studies on the recycling technologies for rare metals mainly by hydrometallurgical methods. Materia Japan., 50(4), 161-167. (in Japanese). https://doi.org/10. 2320/materia.50.161

Yan, G., \& Alstad, J. (1995). Separation of Rhodium from Ruthenium and Iridium by fast solvent extraction with HDEHP. J. Radioanal. Nucl. Chem., 196(2), 287-293. https://doi.org/10.1007/bf02038046

\section{Copyrights}

Copyright for this article is retained by the author(s), with first publication rights granted to the journal.

This is an open-access article distributed under the terms and conditions of the Creative Commons Attribution license (http://creativecommons.org/licenses/by/4.0/). 